\title{
Microencapsulation: its application in nutrition
}

\author{
P. M. M. Schrooyen, R. van der Meer and C. G. De Kruif* \\ NIZO Food Research, PO Box 20, 6710 BA Ede, The Netherlands
}

\begin{abstract}
The development of new functional foods requires technologies for incorporating healthpromoting ingredients into food without reducing their bioavailability or functionality. In many cases, microencapsulation can provide the necessary protection for these compounds, but in all cases bioavailability should be carefully studied. The present paper gives an overview of the application of various microencapsulation technologies to nutritionally-important compounds, i.e. vitamins, $n-3$ polyunsaturated fatty acids, $\mathrm{Ca}, \mathrm{Fe}$ and antioxidants. It also gives a view on future technologies and trends in microencapsulation technology for nutritional applications.
\end{abstract}

Microencapsulation: Functional foods: Bioavailability

Currently, there is a trend towards a healthier way of living, which includes a growing awareness by consumers of what they eat and what benefits certain ingredients have in maintaining good health. Preventing illness by diet is a unique opportunity for innovative so-called functional foods (Hilliam, 1996; Sheehy \& Morrissey, 1998). These products often present new challenges to the food engineer. Existing and new ingredients need to be incorporated into food systems, in which they slowly degrade and lose their activity, or become hazardous by oxidation reactions. Ingredients can also react with components present in the food system, which may limit bioavailability, or change the colour or taste of a product. In many cases, microencapsulation can be used to overcome these challenges.

Microencapsulation is the envelopment of small solid particles, liquid droplets or gases in a coating (Thies, 1987). Microcapsules are small $(1-1000 \mu \mathrm{m})$ and can have many morphologies. In general, one can distinguish between mononuclear capsules, which have one core enveloped by a shell, and aggregates, which have many cores embedded in a matrix. The shell or matrix materials are usually polymers. The many available technologies for microencapsulation can be divided into two categories, one which uses a liquid as a suspending medium (complex coacervation, interfacial and in situ polymerization or solvent evaporation from emulsions) and one which uses a gas as a suspending medium into which a liquid phase is sprayed (spray-drying or spray-cooling, fluidized-bed coating or coextrusion). There are a number of excellent reviews of the various processes for preparing microcapsules (Thies, 1987;
Dziezak, 1988; Jackson \& Lee, 1991; Shahidi \& Han, 1993).

Microcapsules are also used in applications such as carbonless copy paper, pharmaceuticals, pesticides and scented strips. In the food industry the use of microencapsulation to protect, isolate or control the release of a given substance is of growing interest. Converting a liquid into a powder allows alternative use of ingredients. One of the largest food applications is the encapsulation of flavours. A good general overview of encapsulated food ingredients is given by Shahidi \& Han (1993).

The present paper will focus on the use of microencapsulation for nutritional ingredients: vitamins, polyunsaturated fatty acids (PUFA), minerals, antioxidants, new functional ingredients. The influence of the processing technology and matrix materials used on the stability and bioavailability of these ingredients will be discussed.

\section{Vitamins}

Both lipid-soluble (e.g. vitamin A, $\beta$-carotene, vitamins D, $\mathrm{E}$ and $\mathrm{K}$ ) and water-soluble (e.g. ascorbic acid) vitamins can be encapsulated using various technologies. The most common reason for encapsulating these ingredients is to extend the shelf-life, either by protecting them against oxidation or by preventing reactions with components in the food system in which they are present.

A good example is ascorbic acid (vitamin C), which is added extensively to a variety of food products as either an antioxidant or a vitamin supplement (Kirby et al. 1991). Its application as a vitamin supplement is impaired by its high 
reactivity and, hence, poor stability in solution. It can degrade by a variety of mechanisms. Oxidation of ascorbic acid is a free radical-mediated process, which results in a series of reactive intermediates (for review, see Liao \& Seib, 1988). It is catalysed by transition metal ions and is accelerated at neutral to alkaline $\mathrm{pH}$. Furthermore, losses due to enzymic or anaerobic destruction of vitamin $\mathrm{C}$ can occur.

It is a true challenge to encapsulate water-soluble food ingredients to protect them against deterioration during the shelf-life of the food product in which they are used. It is often difficult, using only food-grade ingredients, to achieve a barrier which is good enough to prevent these water-soluble compounds from leaking away into the food system. The most obvious way to encapsulate these compounds is by spray-cooling and spray-chilling. These techniques involve dispersing the water-soluble ingredient in a molten fat or wax and spraying this dispersion through heated nozzles into a chamber at ambient temperature (spray-cooling) or at refrigeration temperatures (spraychilling). If the chamber is at room temperature, the encapsulation material has a melting point between 45 and $122^{\circ} \mathrm{C}$. If the chamber is cooled, materials melting at $32-42^{\circ} \mathrm{C}$ can be used (Thies, 1987). The microcapsules are insoluble in water and will release their contents when the temperature of the food product is raised above the melting temperature of the fat or wax. Thus, a kind of temperaturecontrolled release is achieved. If the fat crystallisation process during spray-cooling or spray-chilling is not properly controlled, unwanted release properties or particle softening can occur.

Another technique which can be used to encapsulate water-soluble ingredients is fluidized-bed coating. Here the dry water-soluble ingredient is suspended in an upwardmoving stream of air, which is temperature and humidity controlled. A molten wax or fat is sprayed onto the particles in order to obtain a coating around them. A review of this technology was given by DeZarn (1995) and by Dewettinck \& Huyghebaert (1999).

For vitamin C encapsulation, both spray-cooling or spraychilling and fluidized-bed coating can be used when the vitamins are added to solid foods, such as cereal bars, biscuits or bread. For application in liquid food systems, the best way to protect water-soluble ingredients is by encapsulation in liposomes. Liposomes are single or multilayered vesicles of phospholipids containing either aqueous-based or lipophilic compounds. A review of their structure and applications in food is given by Reineccius (1995). Application of liposomes in foods is possible if no organic solvents are used, e.g. using the dehydration-rehydration procedure described by Kirby \& Gregoriadis (1984). Kirby et al. (1991) have found that ascorbic acid incorporated in liposomes exhibited a half-life of $100 \mathrm{~d}$ compared with a pure solution of ascorbic acid with a half-life of $18 \mathrm{~d}\left(4^{\circ} \mathrm{C}\right)$. Also, reactions with other components present in food systems may be avoided by inclusion of ascorbic acid in liposomes.

Lipid-soluble vitamins such as vitamin A, $\beta$-carotene and vitamins $\mathrm{D}, \mathrm{E}$ or $\mathrm{K}$ are much easier to encapsulate than water-soluble ingredients. A commonly-used procedure is spray-drying of emulsions (Kowalski et al. 2000). Spraydrying is the industrial method of choice for encapsulation of oil-based compounds such as flavours, vitamins and fatty acids (Ré, 1998). In a typical process all matrix materials, such as gum arabic and maltodextrin, are hydrated in water and the oil-based substance is added while stirring. This mixture is subsequently homogenized to form an emulsion. The emulsion can then be spray-dried to yield a powder. Such a powder typically contains $1-50 \%$ (w/w) oil. Recent patents describe methods to incorporate more lipophilic substances such as vitamins in a powder (Boyle \& Chang, 1999; Stroh et al. 2000). These microencapsulated vitamins are often used to prepare tablets. The type of matrix material used can strongly influence the tablet properties (Stroh et al. 2000) and the oxidation stability of the encapsulated compounds (Schrooyen et al. 2000). The stability against oxidation is related to the $\mathrm{O}_{2}$-permeability of the matrix (Baisier \& Reineccius, 1989; Goubet et al. 1998). The diffusion of gases such as $\mathrm{O}_{2}$ through the capsule wall depends on the state of the polymer mixture composing the capsule. At low water activity and temperature most polymers are in a solid glassy state, and gas diffusion is limited. At higher water activity and temperature, above the so-called glass-transition temperature, materials change into a liquid-like rubbery state, permitting molecular diffusion. Several approaches exist to predict glass-transition temperatures in complex biopolymer mixtures (Gordon \& Taylor, 1952; Couchman \& Karasz, 1978; Matveev et al. 2000). In our laboratory orange-peel oil was encapsulated in four different matrices by emulsion spray-drying: gum arabic, gum arabic-maltodextrin, caseinate-maltodextrin, a plant polysaccharide (Schrooyen et al. 2000). Orange-peel oil mainly consists of limonene, which is easily oxidized. Oxidation products, such as limonene-1,2-epoxide and carvone, can be quantified using GC. In an accelerated shelf-life test $\left(37^{\circ} \mathrm{C}\right)$ it was found that the plant polysaccharide resulted in at least six times less limonene-1,2epoxide than the other matrices, which indicated that $\mathrm{O}_{2}$ transport through this biopolymer film is much slower than that through the other material types. We are currently investigating further the physical properties of this polysaccharide.

De Man et al. (1986) found an increase in vitamin A stability on storage of vitamin A-enriched non-fat dried milk when microencapsulated vitamins were used. The microcapsules used were commercial samples, and no details are given of their preparation method. Wagner \& Warthesen (1995) reported the influence of the addition of various maltodextrins on the stability of $\beta$-carotene in spray-dried carrot juice. The hydrolysed starch was mixed with carrot juice and then spray-dried. Addition of maltodextrins increased the shelf-life of carrot powders up to 220-fold compared with carrot juice spray-dried alone. A higher dextrose equivalent (DE) of the maltodextrins used resulted in better protection. This finding is consistent with the results of a study on the protection by maltodextrins against oxidation of orange oil (Anandaraman \& Reineccius, 1986). High-DE maltodextrins are however hygroscopic, which can result in poor powder properties. Mixing low-DE maltodextrins with glucose, galactose or lactose to obtain a $25 \mathrm{DE}$ maltodextrin resulted in an increased shelf-life of encapsulated trans- $\beta$-carotene when compared with commercial 25 DE maltodextrin (Desobry et al. 1999). 


\section{n-3 Polyunsaturated fatty acids}

$n-3$ PUFA have been identified as essential to human subjects during the whole of their lifetime, as a result of the pioneering research of Bang \& Dyerberg (1972) on the Greenland Eskimos. A high intake of PUFA is associated with a low incidence of CHD and a reduced risk of cancers (Wallace et al. 2000). In a large part of the Western world consumption of fatty fish is too low to meet recommendations by, for example, the British Nutrition Foundation Task Force (1992). Thus, enrichment of food with fish oil has been studied intensively as an acceptable and effective means of increasing the levels of $n$-3 PUFA in the general population. However, $n-3$ PUFA are also prone to oxidation because of the high number of unsaturated double bonds in the fatty acyl chains. This factor limits their shelf-life to 6 months, when stored at $4^{\circ} \mathrm{C}$ in closed containers under $\mathrm{N}_{2}$. Encapsulation by emulsion spray-drying has been used successfully to increase the shelf-life of this type of ingredients, and allows their use in a large variety of foods such as infant formulas and bread mixes (Andersen, 1995). Andersen (1995) reports that a layer of starch is added to the particles while spray-drying in order to prevent them from clinging together during the final drying step. The influence of various process variables on oxidation during the emulsifying and drying stage is not known.

It was demonstrated in two recent studies that consuming food enriched with microencapsulated fish oil obtained by emulsion spray-drying was as effective as the daily intake of fish oil gelatine capsules in increasing dietary levels of n-3 long-chain PUFA (Higgins et al. 1999; Wallace et al. 2000). This finding indicates that the bioavailability of $n-3$ PUFA added to the food matrix is maintained by microencapsulation. It was also found that the shelf-life of the fatty acids could be increased to more than 2 years by microencapsulation (Andersen, 1995). Kolanowski et al. (1999) studied the possibilities of enriching certain food products with n-3 PUFA. Microencapsulated fish oil (ROPUFA '10' $n-3$ eicosapentaenoic acid powder; Roche, Basel, Switzerland) was used to enrich instant powdered-milk-based protein-carbohydrate formulas, flavoured with cocoa or strawberry and without extra flavours. Up to $18 \%(\mathrm{w} / \mathrm{w})$ microencapsulated fish oil was added (1.8\% (w/w) PUFA). Palatability of $3-6 \%(\mathrm{w} / \mathrm{w})$ enriched samples $(0 \cdot 3-0.6 \%$ (w/w) PUFA) stored for 6 months was good throughout the storage time.

\section{Minerals \\ Iron}

From a nutritional point of view $\mathrm{Fe}$ is one of the most important elements, and its deficiency affects about onethird of the world's population. The best way to prevent this problem is through the Fe fortification of food. However, the bioavailability of $\mathrm{Fe}$ is negatively influenced by interactions with food ingredients such as tannins, phytates and polyphenols. Moreover, Fe catalyses oxidative processes in fatty acids, vitamins and amino acids, and consequently alters sensory characteristics and decreases the nutritional value of the food. Microencapsulation can be used to prevent these reactions, although bioavailability should be checked carefully. The bioavailability of readily watersoluble Fe salts such as $\mathrm{FeSO}_{4}$ or ferrous lactate is higher than that of poorly water-soluble (e.g. ferrous fumarate) or water-insoluble (e.g. $\mathrm{FePO}_{4}$ ) Fe. Suitable encapsulation techniques depend on the water solubility of the compound. Liposome technology is the method of choice for $\mathrm{Fe}$ fortification of fluid food products. An Fe bioavailability study on milk enriched with $\mathrm{FeSO}_{4}$ encapsulated in a lecithin liposome has been conducted (Boccio et al. 1997; Uicich et al. 1999). Heat treatment and storage for 6 months did not result in decreased $\mathrm{Fe}$ bioavailability of the Fe-fortified milk. Bioavailability was similar to absorption of Fe from high-bioavailable $\mathrm{FeSO}_{4}$.

\section{Calcium fortification}

Soya milk contains much less Ca $(12 \mathrm{mg} / 100 \mathrm{~g}$ soya milk) than cow's milk (120 mg/100 g cow's milk), which is undesirable from a nutritional point of view. Attempts to fortify soya milk with $\mathrm{Ca}$ salts (calcium triphosphate and calcium citrate) have been unsuccessful because this process causes the soyabean proteins to coagulate and precipitate (Weingartner et al. 1983). By encapsulating the Ca salt (calcium lactate) in a lecithin liposome it was possible to fortify $100 \mathrm{~g}$ soya milk with up to $110 \mathrm{mg} \mathrm{Ca}$, thereby reaching levels equivalent to those in normal cow's milk (Hirotsuka et al. 1984). The soya milk remained stable at $4^{\circ} \mathrm{C}$ for at least 1 week. It would be desirable to know how stable a fortified long-shelf-life soya milk would be, and how the Ca bio-availability of soya milk compares with that of cow's milk.

\section{Antioxidants}

Ascorbic acid is often used as an antioxidant in foods. By putting it in a liposome together with vitamin $\mathrm{E}$, with which ascorbic acid can have a synergistic antioxidant effect, it could be used for protection of emulsion-type foods (Reineccius, 1995). Vitamin E is incorporated into the liposome wall (Berrocal \& Abeger, 1999), whereas the ascorbate is entrapped in the aqueous interior. This system is then used as an emulsifier in an emulsion, and adsorbs at the water-oil interface. In this way the antioxidants are targeted at the site where oxidative reactions generally occur (Pothakamury \& Barbosa-Cánovas, 1995).

\section{Complex coacervation: an old technology for new applications}

Complex coacervation is an interesting encapsulation technology which has been used extensively in non-food and pharmaceutical applications over the past 50 years. The largest application used to be in carbonless copy paper. The phenomenon can occur when two oppositely-charged polymers form a soluble electrostatically-bound complex (Bungenberg De Jong, 1949; Voorn \& Overbeek, 1957). It can be used to encapsulate water-insoluble ingredients to increase shelf-life, to allow alternative processing, to control the release of the encapsulated component or to mask the taste of it (Schmitt et al. 1998). Generally, a protein and a polysaccharide are used to form complexes. Gelatine is 
often used as a protein. Commercially-used polysaccharides are gum arabic and carboxymethylcellulose. Givaudan (Vernier, Switzerland) uses it for special flavours to protect them against shear or temperature. Complex coacervation is usually considered an expensive method for encapsulating food ingredients, but this factor should be related to the benefits it could offer, especially to high-value, labile functional ingredients. Possible nutritional applications could be taste masking of unpleasantly-tasting healthpromoting compounds or protection of, for example, phytosterols against degradation during processing.

Research in our laboratory is focusing on understanding the interactions between biopolymers. For encapsulation purposes we are looking for alternatives to gelatine in complex coacervation. We have developed fast screening tools which can be used to identify the $\mathrm{pH}$ region in which a certain biopolymer combination forms complexes, and what the influence of salt:biopolymer is on the interactions. The use of complex coacervation in the field of functional foods is expected to increase in the near future.

\section{Future trends}

Many companies and research institutes are looking for new ingredients with possible health benefits. Phytochemicals, wood-derived ingredients such as phytosterols, pro- and prebiotics, new types of carotenoids, trace minerals and polyphenols are examples of such ingredients. Many of these ingredients are or will be available in a purified form within the next 10 years. Adding them to food systems will often require technological innovations. Microencapsulation will certainly play an important role in this process, although it will always make an ingredient more expensive to use and bioavailability should always be considered carefully.

\section{References}

Anandaraman S \& Reineccius GA (1986) Stability of encapsulated orange peel oil. Food Technology 40 (11), 88-93.

Andersen S (1995) Microencapsulated omega-3 fatty acids from marine sources. Lipid Technology 7, 81-85.

Baisier W \& Reineccius GA (1989) Spray drying of food flavors $\mathrm{V}$ : factors influencing shelf-life of encapsulated orange peel oil. Perfumer and Flavorist 14, 48-53.

Bang HO \& Dyerberg J (1972) Plasma lipids and lipoproteins in Greenlandic west coast Eskimos. Acta Medica Scandinavica 192, 85-94.

Berrocal MC \& Abeger A (1999) Shelf life of a saturated vitamin E carrier system for use in the food industry (note). Food Science and Technology International 5, 509-513.

Boccio JR, Zubillaga MB, Caro RA, Gotelli CA, Gotelli MJ \& Weill R (1997) A new product to fortify fluid milk and dairy products with high-bioavailable ferrous sulfate. Nutrition Reviews 55, 240-246.

Boyle S \& Chang K-T (1999) Encapsulation of oleophilic substances and compositions produced thereby. US Patent 5925 381.

British Nutrition Foundation Task Force (1992) British Nutrition Foundation Task Force Report: Unsaturated Fatty Acids: Nutritional and Physiological Significance. London: Chapman \& Hall.
Bungenberg De Jong HG (1949) Crystallisation - coacervation flocculation. In Colloid Science, vol. 2, pp. 232-258 [HR Kruyt, editor]. London: Elsevier.

Couchman PR \& Karasz FF (1978) A classical thermodynamic discussion on the effect of composition on glass-transition temperatures. Macromolecules 11, 117-119.

De Man JM, De Man L \& Wygerde T (1986) Stability of vitamin A beadlets in nonfat dry milk. Milchwissenschaft 41, 468-469.

Desobry SA, Netto FM \& Labuza TP (1999) Influence of maltodextrin systems at an equivalent $25 \mathrm{DE}$ on encapsulated $\beta$ carotene loss during storage. Journal of Food Processing Preservation 23, 39-55.

Dewettinck K \& Huyghebaert A (1999) Fluidized bed coating in food technology. Trends in Food Science and Technology 10, 163-168.

DeZarn TJ (1995) Food ingredient encapsulation. In Encapsulation and Controlled Release of Food Ingredients, American Chemical Society Symposium Series no. 590, pp. 113-131 [SJ Risch and GA Reineccius, editors]. Washington, DC: American Chemical Society.

Dziezak JD (1988) Microencapsulation and encapsulated ingredients. Food Technology 42 (4), 136-151.

Gordon M \& Taylor JS (1952) Ideal copolymers and second-order transitions in synthetic rubbers. I. Non-crystalline polymers. Journal of Applied Chemistry 2, 493-500.

Goubet I, Le Quere J-L \& Voilley AJ (1998) Retention of aroma compounds by carbohydrates: influence of their physicochemical characteristics and of their physical state. A review. Journal of Agricultural and Food Chemistry 46, 1981-1990.

Higgins S, Carroll YL, O’Brien NM \& Morrissey PA (1999) Use of microencapsulated fish oil as a means of increasing n-3 polyunsaturated fatty acid intake. Journal of Human Nutrition and Dietetics 12, 265-271.

Hilliam M (1996) Functional foods: the Western consumer viewpoint. Nutrition Reviews 54, 189-194.

Hirotsuka M, Taniguchi H, Narita H \& Kito M (1984) Calcium fortification of soy milk with calcium-lecithin liposome system. Journal of Food Science 49, 1111-1112, 1127.

Jackson LS \& Lee K (1991) Microencapsulation and encapsulated ingredients. Lebensmittel Wissenschaft und Technologie 24, 289-297.

Kirby CJ \& Gregoriadis G (1984) Dehydration-rehydration vesicles: a simple method for high yield drug encapsulation in liposomes. Biotechnology 2, 979-984.

Kirby CJ, Whittle CJ, Rigby N, Coxon DT \& Law BA (1991) Stabilization of ascorbic acid by microencapsulation in liposomes. International Journal of Food Science and Technology 26, 437-449.

Kolanowski W, Swiderski F \& Berger S (1999) Possibilities of fish oil application for food products enrichment with $\omega$-3 PUFA. International Journal of Food Sciences and Nutrition 50, 39-49.

Kowalski RE, Mergens WJ \& Scialpi LJ (2000) Process for manufacture of carotenoid compositions. US Patent 6093348.

Liao M-L \& Seib PA (1988) Chemistry of L-ascorbic acid related to foods. Food Chemistry 30, 313-317.

Matveev YI, Grinberg VY \& Tolstoguzov VB (2000) The plasticizing effect of water on proteins, polysaccharides and their mixtures. Glassy state of biopolymers, food and seeds. Food Hydrocolloids 14, 425-437.

Pothakamury UR \& Barbosa-Cánovas GV (1995) Fundamental aspects of controlled release in foods. Trends in Food Science and Technology 6, 397-406.

Ré MI (1998) Microencapsulation by spray drying. Drying Technology 16, 1195-1236. 
Reineccius GA (1995) Liposomes for controlled release in the food industry. In Encapsulation and Controlled Release of Food Ingredients, American Chemical Society Symposium Series no. 590, pp. 113-131 [SJ Risch and GA Reineccius, editors]. Washington, DC: American Chemical Society.

Schmitt C, Sanchez C, Desobry-Banon S \& Hardy J (1998) Structure and technofunctional properties of proteinpolysaccharide complexes: a review. Critical Reviews in Food Science and Nutrition 38, 689-753.

Schrooyen P, De Ruiter G \& De Kruif C (2000) Spray-dried orange oil emulsions: influence of cold water dispersible matrices on retention and shelf life. Proceedings of the International Symposium on the Controlled Release of Bioactive Materials, pp. 1317-1318. Deerfield, IL: Controlled Release Society, Inc.

Shahidi F \& Han X-Q (1993) Encapsulation of food ingredients. Critical Reviews in Food Science and Nutrition 33, 501-547.

Sheehy PJA \& Morrissey PA (1998) Functional foods: prospects and perspectives. In Nutritional Aspects of Food Processing and Ingredients, pp. 45-65 [CJK Henry and NJ Heppell, editors]. Gaithersburg, MD: Aspen Publishers.

Stroh F, Bower D, Chaundy F, Finnan J \& Soblesky T (2000) Method of making spray-dried powders with high edible-oil loadings based on non-hydrolyzed gelatin. US Patent 6020003 .
Thies C (1987) Microencapsulation. In Encyclopedia of Polymer Science and Engineering, pp. 724-745 [HF Mark, NM Bikales, CG Overberger, G Menges and JI Kroschwitz, editors]. New York: John Wiley \& Sons.

Uicich R, Pizarro F, Almeida C, Diaz M, Bocchio J, Zubillaga M, Carmuega E \& O'Donnell A (1999) Bioavailability of microencapsulated ferrous sulfate in fluid cow's milk. Studies in human beings. Nutrition Research 19, 893-897.

Voorn JTG \& Overbeek MJ (1957) Phase separation in polyelectrolyte solutions. Theory of complex coacervation. Journal of Cellular and Comparative Physiology 49, Suppl. 1, 7-26.

Wagner LA \& Warthesen JJ (1995) Stability of spray-dried encapsulated carrot carotenes. Journal of Food Science 60, 1048-1053.

Wallace JMW, McCabe AJ, Robson PJ, Keogh MK, Murray CA, Kelly PM, Marquez-Ruiz G, McGlynn H, Gilmore WS \& Strain JJ (2000) Bioavailability of $n-3$ polyunsaturated fatty acids (PUFA) in foods enriched with microencapsulated fish oil. Annals of Nutrition and Metabolism 44, 157-162.

Weingartner KE, Nelson Al \& Erdman JW Jr (1983) Effects of calcium addition on stability and sensory properties of soy beverage. Journal of Food Science 48, 256-257, 263. 